\title{
MODELS OF IMAGES WITH RADIAL-CIRCULAR STRUCTURE
}

\author{
V.R. Krasheninnikov ${ }^{1}$, O.E. Malenova ${ }^{1}$, A.U. Subbotin ${ }^{1}$ \\ ${ }^{1}$ Ulyanovsk State Technical University, Ulyanovsk, Russia - o.malenova@ulstu.ru
}

\section{Commision II, WG II/5}

KEY WORDS: Image, Random Field, Radial-Circular Structure, Model, Simulation, Doubly Stochastic

\begin{abstract}
:
The overwhelming majority of known image models are varieties of random fields defined on rectangular two-dimensional grids or grids of higher dimension, for example. In some practical situations, the images have an annular, radial or radial-circular structure. For example, images of the facies (thin film) of dried biological fluid, eyes, cut of a tree trunk or a fruit, blood vessel, erythrocyte, blast pattern, end face detail, etc. In addition, radar and other images are physically obtained in polar or spherical coordinates. These features of images require their consideration in their mathematical models. In this paper, an autoregressive models of homogeneous and inhomogeneous random fields defined on a circle or oval are considered as representations of images with radial or radialcircular structure.
\end{abstract}

\section{INTRODUCTION}

At present, the overwhelming majority of known image models are varieties of random fields defined on rectangular twodimensional grids or grids of higher dimension (Duda et al., 2000, Gonzalez et al., 2017, Jähne, 2005, Pratt, 2001). Among these models there are autoregressive, polynomial, Gibbs, canonical decompositions, and so on (Gimel'farb, 1999, Soifer, 2009, Vizilter et al., 2015, Woods, 1981). There are also works on fields defined on a sphere and other curved surfaces (Krasheninnikov et al., 2017). In some practical situations, the images have a circular, radial or radial-circular structure. For example, images of the facies (thin film) of dried biological fluid (Figure 1, a), an eye (Figure 1, b), a virus (Figure 1, c), cut of a tree trunk (Figure 1, d), sunflower (Figure 1, e), fireworks (Figure 1, f), etc. In addition, radar and other images are physically obtained in polar or spherical coordinates. These features of images require their consideration in random field models, which is necessary for the formulation and solution of problems of processing such images.

Let's consider, for example Habibi model (Habibi, 1972) with autoregressive equation on rectangular grid

$$
x_{k, l}=a x_{k, l-1}+b x_{k-1, l}-a b x_{k-1, l-1}+c \xi_{k, l},
$$

where $\quad x_{k, l}$ is a value at node $(k, l)$,

$\xi_{k, l}$ are independent standard random variables.

The covariance function $(\mathrm{CF})$ of this model is

$$
V(m, n)=M\left(x_{k, l} x_{k+m, l+n}\right)=\sigma^{2} a^{|n|} b^{|m|},
$$

where $\sigma^{2}=c^{2} /\left(\left(1-a^{2}\right)\left(1-b^{2}\right)\right)$ is the variance. The isocovariation lines $V(m, n)=$ Const are rhombus.

If a circle is cut from some two-dimensional image, then a radial-circular structure will not be desirable in the resulting image. Therefore, these images require special models.

\section{AUTOREGRESSIVE MODEL ON A CIRCUL}

Let's consider the cylindrical model that will be the base for the circular image. If a cylindrical image (for example, image of a rotation shaft) is cut lengthwise and expanded, then it is transformed into a rectangular image. Points along the cross sections are close to each other on the original cylindrical image that is why their values are highly correlated. In the cut image, these points are located at the opposite ends. Thus, the line ends are highly correlated. However, such images cannot be described by usual models on the rectangles because the correlations in these models are weakening, when the distances between pixels increases. Thus, the image points at the line ends do not possess the necessary high correlation.

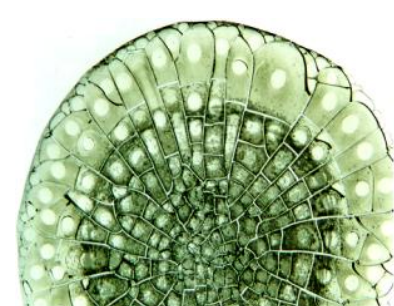

a)

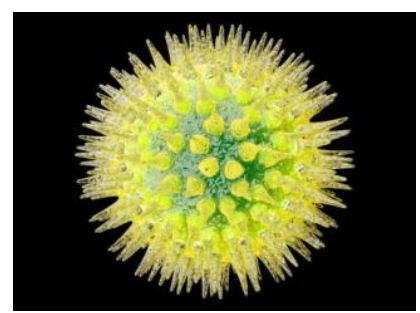

c)

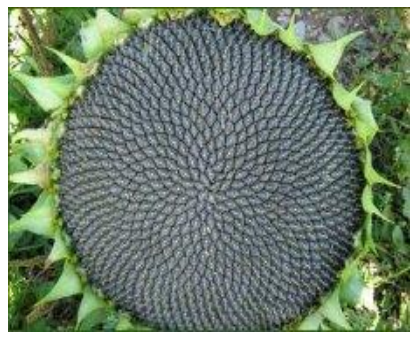

e)

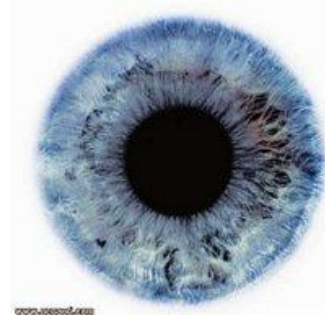

b)

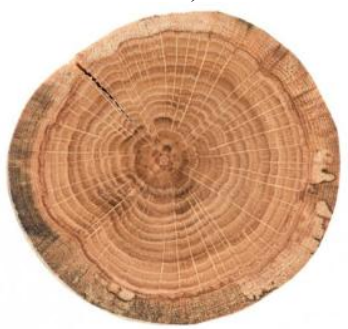

d)

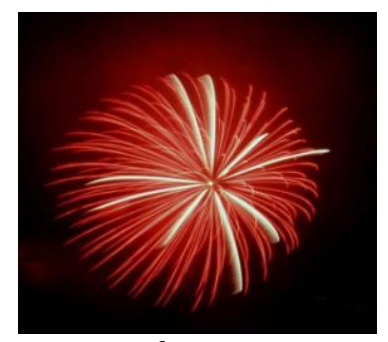

f)
Figure 1. Examples of images with a radial-circular structure: a) facies, b) an eye, c) a virus, d) cut of a tree trunk, e) sunflower, f) fireworks 
Let's consider, for example, an image (Figure 2) simulated by Habibi model (1). This figure shows that the first and the last columns of the rectangular image are significantly different. Thus, when pasting this image into a cylinder, one will observe great jumps in brightness at the junction.

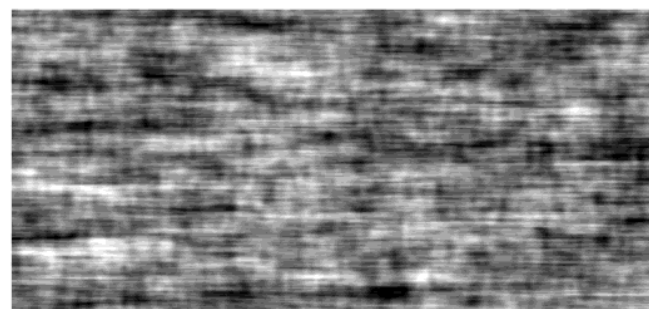

a)

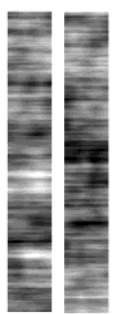

b)
Figure 2. Simulated images: a) image simulated by Habibi model $(1), b)$ the first and the last five columns of this image

To approximate the model to real cylindrical images, consider a spiral grid on the cylinder shown in Figure 3. Grid lines represent spiral turns. To describe an image set on this grid, let us apply the analog (Krasheninnikov et al., 2001) of Habibi model

$$
x_{k, l}=a x_{k, l-1}+b x_{k-1, l}-a b x_{k-1, l-1}+c \xi_{k, l}
$$

where $\mathrm{k}$ is a spiral turn number;

1 is a node number $l=0, \ldots, T$;

$x_{k, l}=x_{k+1, l-T}$ when $l \geq T$;

$T$ is the period, i.e. the number of points in one turn;

$\xi_{k, l}$ are independent standard random variables.

The next value on the turn of this spiral depends on the immediately previous one and on the values from the previous turn. It should be pointed out that in model (3) the grid can be also regarded a simple cylindrical grid, i.e. as a sequence of circles.

This model can be represented in an equivalent form

$$
x_{n}=a x_{n-1}+b x_{n-T}-a b x_{n-T-1}+c \xi_{n},
$$

(where $n=k T+l$ ) as a model of a random process, which is an image scan along the spiral.

Obviously, if $b$ value is close to 1 , then the neighboring image lines (spiral turns) will be slightly different from each other. Thus, this model can be used to describe and simulate cylindrical images.

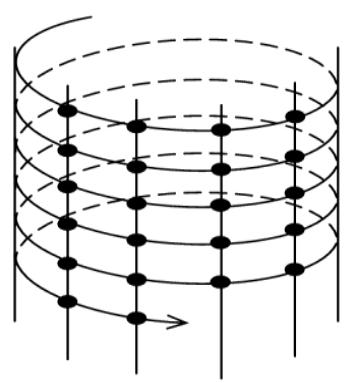

Figure 3. Spiral grid of a cylindrical image

The scanning of image implementation obtained by use of model (4) is shown in Figure 4, a. It is obvious that the values at the line ends are strongly correlated (Figure $4, b$ ), as it should be for a cylindrical image, obtained by image pasting as in Figure 4, a.

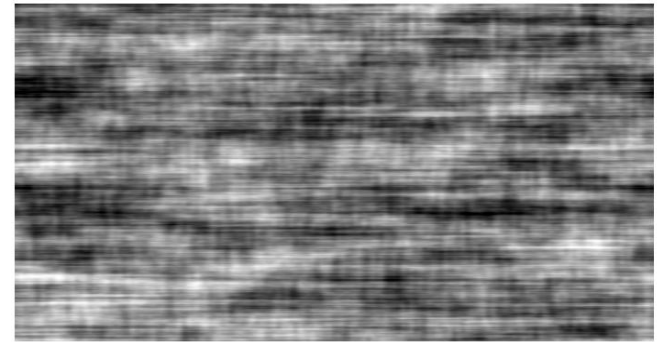

a)

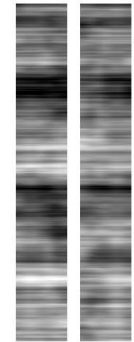

b)
Figure 4. Simulated images: a) image simulated by model (4), b) the first and the last five columns of this image

The characteristic equation of model (4) is

$$
z^{T+1}-a z^{T}-b z+a b=0
$$

or

$$
\left(z^{T}-b\right)(z-a)=0
$$

therefore, the $\mathrm{CF}$ of random process $\left\{x_{n}, n=0,1, \ldots\right\}$ is expressed through the roots of this equation. as follows

$$
\begin{aligned}
& V(n)=M\left[x_{i} x_{i \pm n}\right]= \\
& c^{2}\left(\frac{1}{\left(1-b^{2}\right) T} \sum_{k=0}^{T-1} \frac{z_{k}}{\left(1-a z_{k}\right)\left(z_{k}-a\right)} z_{k}^{n}+\frac{s}{\left(1-a^{2}\right)(1-b s)(s-b)} \rho^{n}\right)
\end{aligned}
$$

where $z_{k}=\sqrt[T]{b} \exp (i 2 \pi k / T)$ and $s=a^{T}$. In particular, when $n=k T$ we obtain

$$
V(k T)=\frac{c^{2}}{\left(1-a^{2}\right)\left(1-b^{2}\right)(1-s b)(b-s)}\left(\left(1-s^{2}\right) b^{k+1}-\left(1-b^{2}\right) s^{k+1}\right)
$$

and the variance, when $k=0$ :

$$
\sigma^{2}=\frac{c^{2}(1+b s)}{\left(1-a^{2}\right)\left(1-b^{2}\right)(1-b s)}
$$

To reduce the calculations, it is possible to calculate only $V(0)$, $V(1), \ldots, V(T)$ by formula (5), and for the rest of values, use recurrent formula

$$
V(n)=a V(n-1)+b V(n-T)-a b V(n-T-1) .
$$

View of the graph of normalized covariance function (NCF) is shown in Figure 5. The correlation decreases with increasing distance $n$, but at distances divisible by period $T$, it is high.

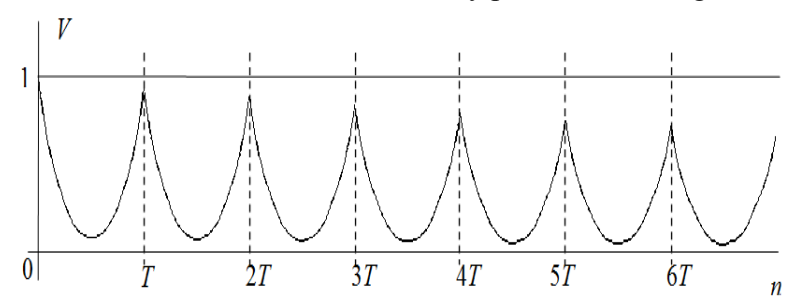

Figure 5. The graph of NCF 
Now consider view of NCF $C(m, n)=M\left[x_{k \pm m} x_{l \pm n}\right] / \sigma^{2}$ on a cylindrical image. The values of this function and its isocorrelation lines $C(m, n)=$ Const are shown in Figure 6 . The lighter areas correspond to larger values. Isolines are shown in black. Figure 6, a shows the values of NCF relative to the central pixel of this figure. Each column corresponds to one turn, that is, values $C(m, n), n=-T / 2, \ldots, 0, \ldots, T / 2$ are shown. It is noticeable that at short distances the NCF isolines are close to rhombuses, which is typical property of Habibi model, in which all isolines are rhombuses. As the distance increases, the isolines are bent. Figure 6, b shows the NCF relative to the central pixel of the lower border of this figure (it is marked with a short line). Each column corresponds to one turn, that is, values $C(m, n), n=0,1, \ldots, T$ are shown. Noticeable that $C(m, n) \approx C(m, n+T)$.

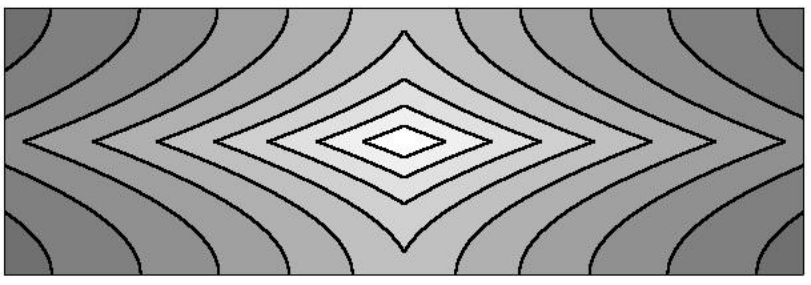

a

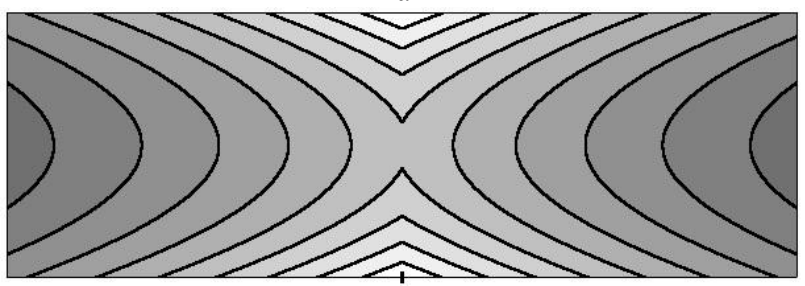

b

Figure 6. Normalized correlation function of cylindrical image: a) relative to the central pixel, b) relative to the lower border.

A polar coordinate system $(r, \varphi)$ is convenient for circular images representation. To do this, we will consider the turns of the cylindrical spiral of model (3) as turns of a circle. In other words, index $k$ is converted into a polar radius, and index $l$ into a polar angle. Thus, the value $x_{k, l}$ in the pixel $(k, l)$ of the cylindrical image is converted to the same value in the pixel $(k \Delta r, l \Delta \varphi)$ of the circular image (Figure 7, a). When using model (4), it is also convenient to use a spiral grid (Figure 5, b), similar to the cylindrical spiral in Figure 3.

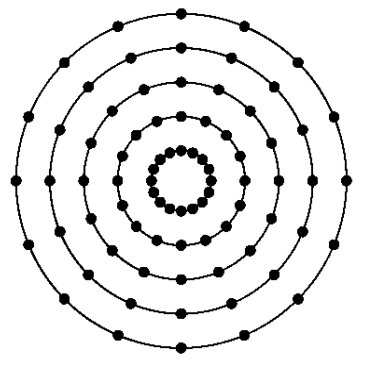

a

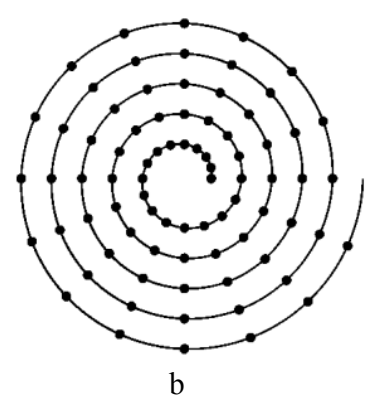

Figure 7. Grids on a circle: a) circular, b) spiral

The parameters $a$ and $b$ of model (3) set the degree of correlation in the radial and circular direction. If the autoregressive coefficient $a$ of the previous pixel is relatively large, then the image will have a high circular correlation (Figure 8). If the autoregression coefficients $b$ from the previous coil of the spiral prevail, the image will be more correlated in the radial direction (Figure 9). If $a \approx b$ then the image is equally correlated in both directions (Figure 10).

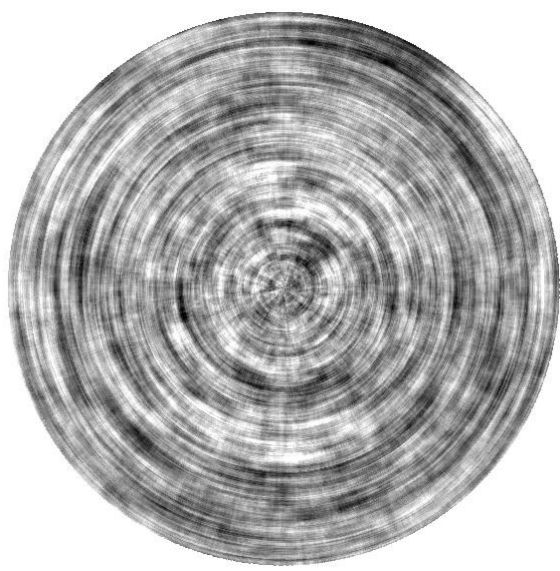

Figure 8 . The image with circular correlation

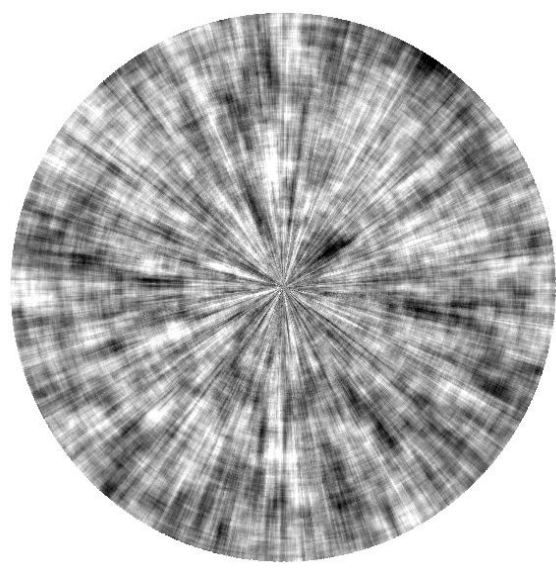

Figure 9. The image with radial correlation

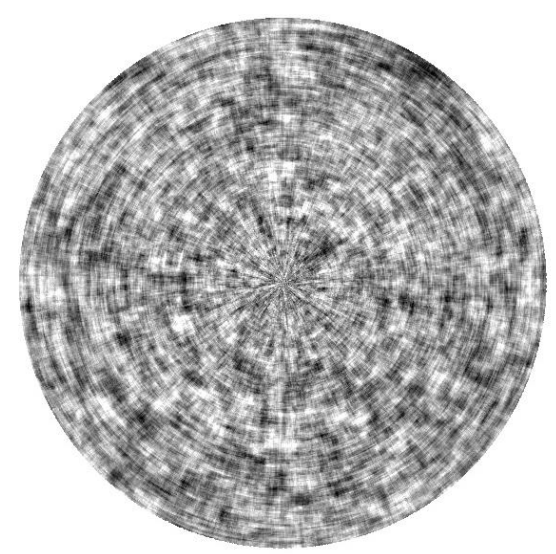

Figure 10. The images with uniform correlation

Let's consider the NCF view of circular image. The circular image in this model is actually a geometric transformation of a cylindrical one, so its NCF can be obtained from the NCF of a cylindrical image. Figure 11, a shows the values of NCF relative to the central pixel of this figure. It is natural that the isolines are circles. Figure 11, b shows the values of NCF and its isocorrelation lines relative to the pixel in the middle of a radius (maximum brightness). Isolines at short distances are close to rhombuses, as in Figure 6, a. 


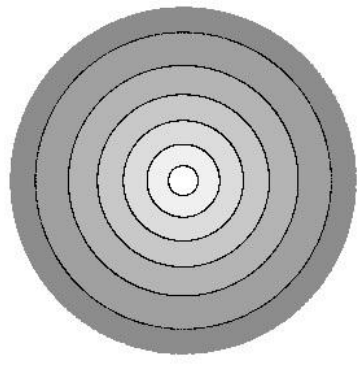

a

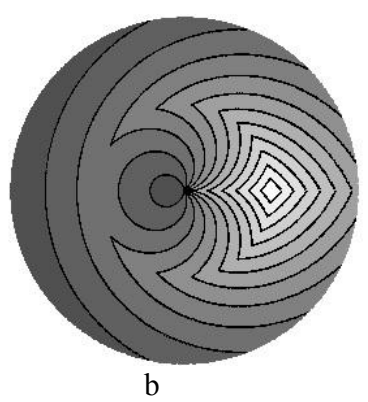

b

Figure 11. Normalized correlation function of circular image: a) relative to the center, $b$ ) relative to the middle of a radius

The images represented by the models described are uniform. In [10], doubly stochastic models were used to represent inhomogeneous images with random inhomogeneities. In these models, some random field sets the parameters of the resulting random field. The same method can be used to represent inhomogeneous circular images. Figure 12 shows the process of forming such an image. Figure 12, a shows a simulated control image. Its threshold section is shown in Figure 12, b. Dark areas correspond to low brightness of image (a). Image 12, a sets the autoregression coefficients of the resulting image 12, c. High brightness of the image 12, a correspond to large values of the autoregression coefficient $a$ in (3) along a circul, and small brightness sets large values $b$ along a radius. As a result, on the resulting image there are areas with a predominance of circular or radial correlation.

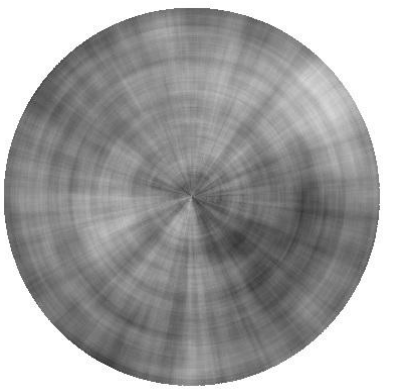

a)

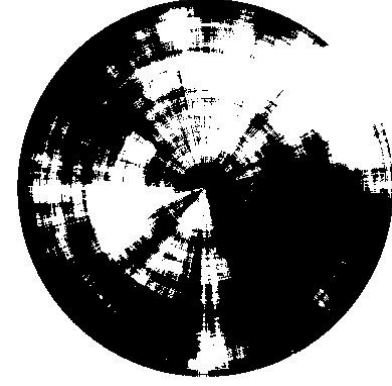

b)

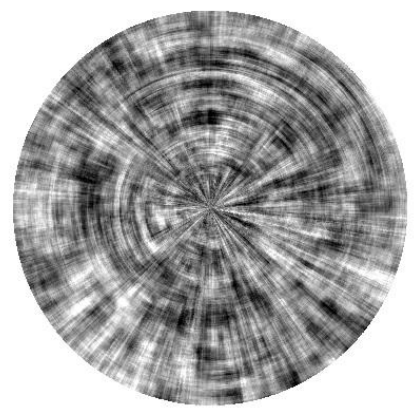

c)

Figure 12. Simulation of inhomogeneous images: a) the control image, b) its threshold section, c) the resulting image

The images in Figure 1, $\mathrm{d}$ and Figure 1, $\mathrm{f}$ have only approximately a radial-circular structure. To represent such images, random fluctuations of the polar radius and angle can be introduced into the model (3). Figure 13, a shows an image with a random angle fluctuation with a regular change in radius. In this case, the overall shape of the image is circular. In Figure $13, \mathrm{~b}$ the radial coordinate is also random. As a result, the image is generally different from the circle.

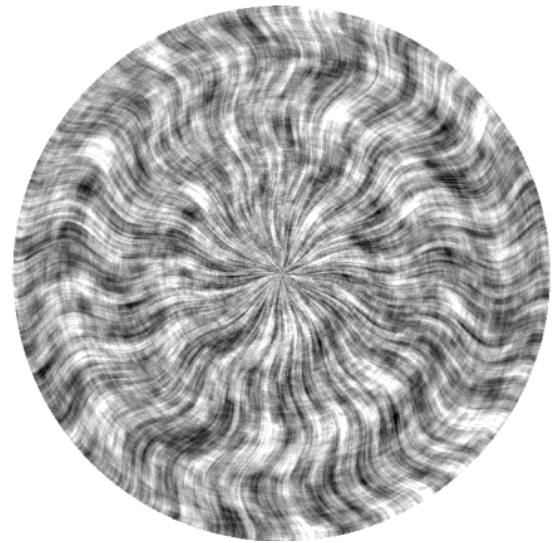

a)

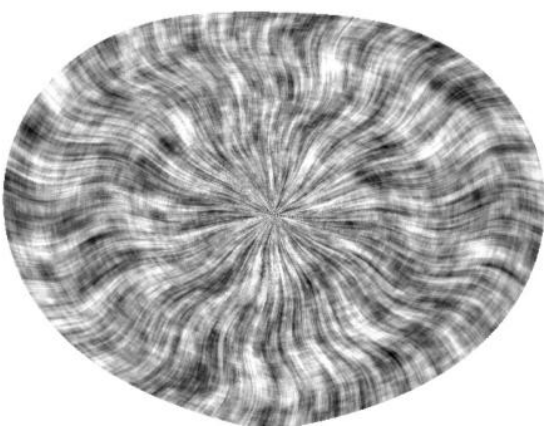

b)

Figure 13. Simulation of deformed images: a) random angle, b) random angle and radius

\section{IDENTIFICATION AND FILTRATION}

For convenience, we assume that the circular image is converted into a sequence. Let the observed image $Z=\left\{z_{n}\right\}$ be an additive mixture of the informative image $X=\left\{x_{n}\right\}$ defined by autoregressive model (4) and white Gaussian noise $\Theta=\left\{\theta_{n}\right\}$ :

$$
z_{n}=x_{n}+\theta_{n}
$$

The parameters of the model (4) and noise dispersion $\sigma_{\theta}^{2}$ in observation model (6) are unknown and, possibly, vary. In the latter case, this variation is assumed to be fairly smooth. It is required to evaluate the informative image $X$ using observations $Z$. To solve this problem, we apply following adaptive pseudogradient analogue of Kalman filter (Krasheninnikov et al., 2017):

$$
\begin{aligned}
& \hat{x}_{n}=\tilde{x}_{n}+s_{n}\left(z_{n}-\tilde{x}_{n}\right)=\tilde{x}_{n}+s_{n} \Delta_{n}, \\
& \tilde{x}_{n}=\rho_{n} \widehat{x}_{n-1}+r_{n} \widehat{x}_{n-T}-\rho_{n} r_{n} \widehat{x}_{n-T-1},
\end{aligned}
$$

where $\tilde{x}_{n}$ is the extrapolated estimate and $\hat{x}_{n}$ is the corrected estimate. Variable parameter vector $\bar{\alpha}_{n}=\left(\rho_{n}, r_{n}, s_{n}\right)$ of this algorithm is calculated using a pseudo-gradient procedure

$$
\bar{\alpha}_{n+1}=\bar{\alpha}_{n}-\mu_{n} \bar{\beta}_{n}
$$

where $\bar{\alpha}_{n+1}$ is the next vector approximation after $\bar{\alpha}_{n}$;

$$
\bar{\beta}_{n}=\nabla\left[\Delta_{n}^{2}\right]=\nabla\left[\left(z_{n}-\tilde{x}_{n}\right)^{2}\right]
$$


is pseudo-gradient of the quality functional $J\left(\bar{\alpha}_{n}\right)=M\left[\Delta_{n}^{2}\right]=M\left[\left(z_{n}-\widetilde{x}_{n}\right)^{2}\right]$, that is, a random vector, the mean of which makes an acute angle with the gradient $\nabla J\left(\bar{\alpha}_{n}\right)$; $\mu_{n}$ is a sequence of positive coefficients affecting values of the steps of the procedure. Thus,

$$
\begin{aligned}
& \rho_{n+1}=\rho_{n}+h_{n} \Delta_{n}\left(\hat{x}_{n}-r_{n} \hat{x}_{n-T-1}\right), \\
& r_{n+1}=r_{n}+h_{n} \Delta_{n}\left(\hat{x}_{n-T}-\rho_{n} \hat{x}_{n-T-1}\right), \\
& S_{n+1}=s_{n}+h_{n} \Delta_{n} \Delta_{n-1},
\end{aligned}
$$

Figures 13 show examples of the application of the described algorithm. Figure 13 (top left) shows an image simulated using model (4) with parameters $a=0.95, b=0.995, T=800$ and unit variance $\sigma_{X}^{2}=1$. This image has a predominantly radial structure. In Figure 13 (top right) is an image of a ring structure with parameters $a=0.995, b=0.95, T=800, \sigma_{X}^{2}=1, \sigma_{\theta}^{2}=1$. These images were distorted by white Gaussian noise with variance $\sigma_{\theta}^{2}=1$ (the middle of Figure 13). The result of the filtering is shown in the bottom of Figure 13. In both examples, the variance of filtering errors was $\sigma_{\varepsilon}^{2} \approx 0,065$, that is, as a result of processing the noise/signal ratio decreased by about 16 times.

Note that during the filtering process the image model is also identified, since the procedures (11) actually estimate the model parameters (4).
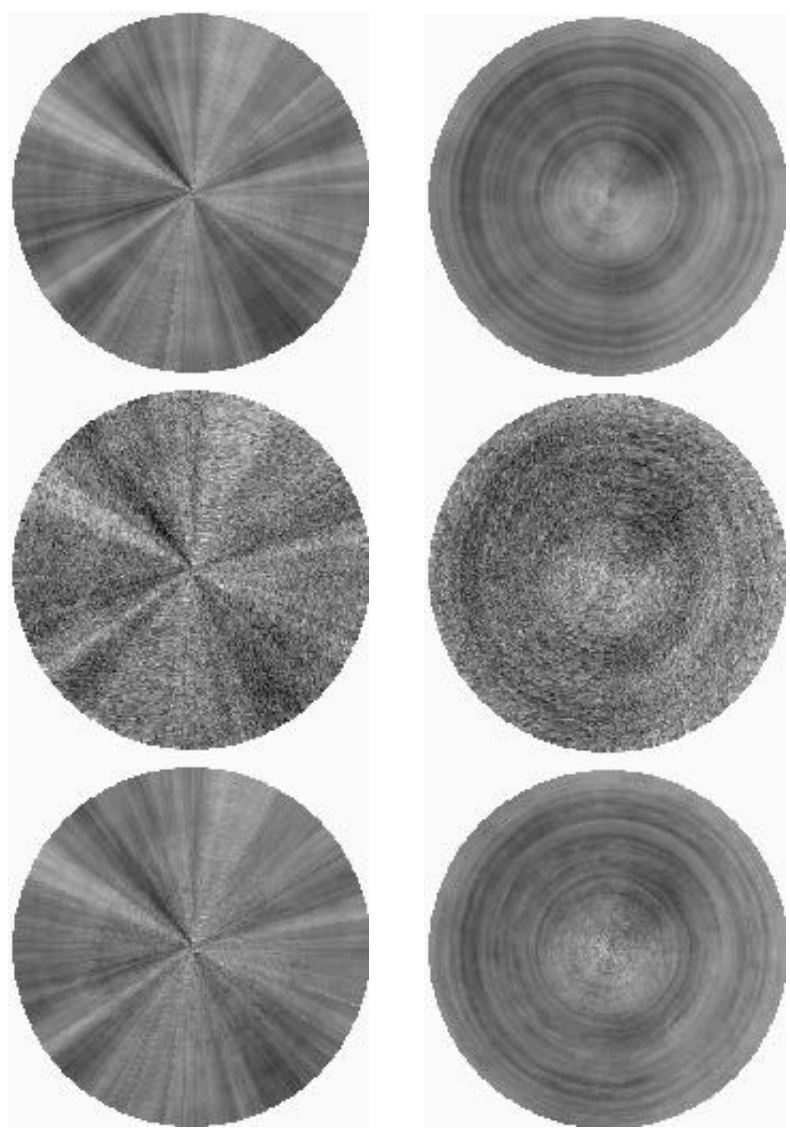

Figure 13. Filtration of circular images: (top) informative images, (middle) noisy images, (bottom) filtered images

\section{CONCLUSIONS}

This paper presented autoregressive models of circular images that have a radial, circular or radial-circular structure. These models are obtained on the basis of autoregressive models of cylindrical images. By varying the parameters of these models, it is possible to obtain images with a predominance of an annular or radial structure. Expressions and graphs of the covariance function of these images are obtained. Models of homogeneous and inhomogeneous images are presented. Heterogeneity is achieved by randomly changing the parameters of the model. An adaptive pseudogradient filtering algorithm for circular images is presented. This algorithm, along with filtering, allows you to identify the image model, that is, to evaluate its parameters.

\section{REFERENCES}

Duda, R.O., Hart, P.E., Stork, D.G., 2000. Pattern classification. 2nd ed. Wiley-Interscience, New York.

Gimel'farb, G.L., 1999. Image Textures and Gibbs Random Fields. Kluwer Academic Publishers Dordrecht.

Gonzalez, R.C., Woods, R.E., 2017. Digital Image Processing. 4th ed. Pearson/Prentice-Hall, New York.

Habibi, A., 1972. Two-dimensional Bayesian estimate of images. Proc IEEE 60(7), pp 878-883.

Jähne, B., 2005. Digital image processing. 6th ed. Springer, Berlin Heidelberg.

Krasheninnikov, V.R., Vasil'ev, K.K., 2017. Multidimensional Image models and processing. Intelligent Systems Reference Library, Vol. 135, pp. 11-64.

Krasheninnikov, V.R., Kalinov, D.V., Pankratov, Yu. G., 2001. Spiral autoregressive model of a quasi-periodic signal. Pat Rec Im An 8(1), pp. 211-213.

Pratt, W.K., 2001. Digital image processing. PIKS Inside. 3rd ed. Wiley, New York.

Soifer, V.A. (Ed), 2009. Computer image processing. Part I: Basic concepts and theory. VDM Verlag Dr. Muller e.K.

Vasil'ev, K.K., Dement'ev ,V.E., Andriyanov, N.A., 2015. Doubly stochastic models of images. Pat Rec Im An 25(1), pp. $105-110$

Vizilter, Y.V., Pyt'ev, Y.P., Chulichkov, A.I., Mestetskiy, L.M., 2015. Morphological image analysis for computer vision applications. In: Favorskaya MN, Jain LC (Eds) Computer Vision in Control Systems-1, ISRL, vol. 73, 9-58. Springer International Publishing Switzerland

Woods, J.W., 1981. Two-dimensional Kalman filtering. In: Huang TS (Ed) Two-Dimensional Digital Signal Processing I: Linear Filters. TAP, Vol. 42, pp. 155-205. Springer Berlin Heidelberg, New York 